\title{
Epigenética: la relación del medio ambiente con el genoma y su influencia en la salud mental.
}

\author{
Epigenetics: The relationship of the environment and mental health. \\ Sandro Casavilca-Zambrano ${ }^{1, a ; 2, b}$, Karina Cancino-Maldonado ${ }^{2, b}$, Luis Jaramillo-Valverde ${ }^{3, b}$, \\ Heinner Guio 1,a; 3,c
}

\section{RESUMEN}

El estudio del genoma humano, cuando efectuado solo a través de la información secuencial de ADN, no explica del todo el alto nivel de variación inter-individual usualmente observado. Es la epigenética la que permite explicar aquellas variaciones de expresión génica como un proceso reversible y hereditario en el corto plazo, bajo la influencia del medio ambiente durante diversas etapas del desarrollo y la edad adulta, sin modificar la secuencia genética. Las alteraciones epigenéticas están siendo ya incorporadas como elementos valiosos en la posible identificación de biomarcadores. Además, debido a su naturaleza reversible, pueden constituirse en factores de mejoría de síntomas de enfermedad mediante el uso de enfoques terapéuticos. El presente artículo explica los mecanismos de inhibición de la expresión génica, su relación con el medio ambiente, la dieta y su influencia en la evolución, la ocurrencia de enfermedades, la conducta humana y la salud mental.

PALABRAS CLAVE: Epigenética, expresión génica, evolución, medio ambiente, salud mental.

\section{SUMMARY}

The study of the human genome, when performed only through the DNA sequence information, does not completely explain the high level of inter-individual variation usually observed. It is the control of gene expression by the epigenetics what allows to explain those variations of gene expression, as a reversible and hereditary process in the short term under the influence of the environment during various stages of development and adulthood, without modifying the genetic sequence. Epigenetic alterations are already being studied as valuable candidates in the eventual identification of biomarkers. Furthermore, their reversible nature makes them promising factors in the amelioration of disease symptoms through the use of therapeutic approaches. This article explains the epigenetic mechanisms and its relationship with the environment, diet, and its influence on evolution, the occurrence of diseases, human behavior and mental health.

KEYWORDS: Epigenetics, gene expression, evolution, environment, mental health.

\footnotetext{
Facultad de Psicología, Universidad de Huánuco. Huánuco, Perú.

2 Instituto Nacional de Enfermedades Neoplásicas, Banco de Tejidos Tumorales. Lima, Perú.

3 INBIOMEDIC Research and Technological Center. Lima, Perú.

a Profesor Investigador; ${ }^{b} \mathrm{MSc},{ }^{\mathrm{c}} \mathrm{PhD}$
} 
Epigenética: la relación del medio ambiente con el genoma y su influencia en la salud mental.

\section{INTRODUCCIÓN}

El Proyecto Genoma Humano se completó en al año 2003 y condujo a la identificación de todos los genes humanos (1); sin embargo, es un hecho que incluso en el caso de gemelos idénticos que comparten secuencias de ADN idénticas, pueden existir diferencias observables en la talla, el peso, el desarrollo de enfermedades, el coeficiente intelectual o incluso el carácter. La clave de este misterio está en la forma cómo los genes se expresan (2). La epigenética puede proporcionar muchas respuestas sobre cómo funcionan los genes y cómo se regulan (3). La naturaleza reversible de los cambios epigenéticos ha atraído el interés en explorar su potencial como objetivos para el desarrollo de tratamientos médicos en personas sanas o portadoras de alguna patología. Más aún, la identificación de efectos secundarios en células humanas sanas es necesaria para mejorar no solo la seguridad del paciente, sino también para apoyar el desarrollo futuro de fármacos evitando la "inestabilidad genómica", como resultado posterior a las modificaciones epigenéticas inducidas por fármacos (4). La epigenética ha tenido un gran desarrollo en la investigación oncológica por haberse reportado su influencia en la proliferación y diferenciación celular $(5,6)$.

Aunque los análisis genéticos clásicos y las tecnologías "omics" (transcriptómica, proteómica, metabolómica, etc.) seguirán proporcionando información importante, también puede resultar información nueva e inesperada del estudio de procesos epigenéticos (metilación del ADN, modificaciones de las histonas, interferencia del ARN). Estos procesos epigenéticos no cambian la secuencia del propio ADN, sino que modifican la forma en que se expresan los genes durante el desarrollo. Se plantea que, se debe ser consciente que el medio ambiente puede tener un fuerte impacto en las etapas de desarrollo posteriores e incluso en la edad adulta; por lo tanto, se requieren estudios adicionales para ayudar a descifrar los mecanismos involucrados (7). Joannes y colaboradores describen el gran potencial y los posibles escollos de la epigenómica poblacional, donde se evidencia el alto nivel de variación epigenómica observado entre individuos dentro de las poblaciones y entre diferentes regiones genómicas $(8,9)$.

\section{El fenotipo}

En 1975, Arthur Rigg, propone a la metilación como el mecanismo de inactivación del cromosoma
$\mathrm{X}$ en mamíferos, siendo este mecanismo descrito en bacterias, el responsable de ciertos eventos permanentes en las células eucarióticas (10). Es el mismo año, Holliday y Pugh plantean que el fenotipo del organismo depende del genotipo y la contribución genética de ambos progenitores planteándose, por primera vez, que la información debería estar escrita en la secuencia de ADN (11). También está claro que los componentes citoplasmáticos pueden tener una poderosa influencia en la expresión génica y el desarrollo. Las células de un organismo multicelular son genéticamente homogéneas pero estructural y funcionalmente heterogéneas, debido a la diferente expresión de los genes. Muchas de estas diferencias en la expresión génica se dan durante el desarrollo y son retenidas posteriormente a través de la mitosis. Las alteraciones estables de este tipo se denominan epigenéticas, ya que son heredables en el corto plazo, pero no implican mutaciones del propio ADN (12).

El proceso de inhibición de la transcripción génica se pueda dar a partir de adición de grupos metilos (metilación) en citosinas localizadas en las islas CpG o CG, acetilación de histonas o la influencia de microRNAs (13). Aprovechando las recientes tecnologías de secuenciación masiva, comenzaron a aparecer reportes en los que se compara el perfil de metilación del ADN con el perfil completo de microRNAs expresados (miRNAome) y la transcriptómica (14).

Por otro lado, el ciclo de la metilación está constituido por cuatro rutas metabólicas que están interrelacionadas: 1) el ciclo de la metionina, 2) el ciclo del folato, 3) el ciclo de la urea y 4) el ciclo BH4 (biopterina). El ciclo de metilación participa de diferentes procesos biológicos como en la reparación de ADN y ARN, procesos inflamatorios, alteraciones en procesos digestivos, silenciamiento del ADN, equilibrio de neurotransmisores, detoxificación de metales, fluidez de membranas celulares, producción de energía (mitocondrias), actividad proteica, mielinización, cáncer, fallo renal, autismo, alzheimer, esquizofrenia, diabetes, fatiga crónica, convulsiones y hasta problemas para dormir (15).

La metilación no modifica el genoma, las modificaciones postraduccionales de las proteínas histonas son mucho más complejas y afectan a múltiples residuos (Arg, Lys, Pro, Ser) en más de 30 sitios dentro de las colas N-terminales de histonas $(16,17)$. La metilación de histonas solo puede aparecer en forma de mono-, di- y tri-metilación; incluso más 
complejas, estas diferentes formas de metilación pueden ocurrir en diferentes residuos de aminoácidos que están situadas en diferentes posiciones (por ejemplo: H3 (Lys 4, 9, 27, 36, 79; Args 2, 17, 16) (18). Varios estudios han demostrado que la metilación del ADN y la desacetilación de las histonas pueden regular la expresión génica sinérgicamente a través de mediadores de proteínas tales como el metil-CpG MeCP2 (19,20). En este sentido realizamos una búsqueda en PUBMED, con el objetivo de revisar la evidencia publicada respecto a cómo el medio ambiente y el entorno familiar, la dieta, y algunas sustancias nocivas pueden influir sobre la salud mental, proponiendo a la epigenética como el mecanismo que influiría en la manifestación de diversas alteraciones y enfermedades.

\section{La evolución}

Los mecanismos epigenéticos también pueden desempeñar un papel importante en los procesos ecológicos y evolutivos (21). Recientemente, los análisis de todo el genoma han demostrado que los niveles actuales de metilación del ADN pueden predecirse a partir de las firmas evolutivas de la metilación delADN, lo queindica que estos dos procesos están íntimamente correlacionados. Curiosamente, los análisis comparativos de los seres humanos y especies de primates estrechamente relacionados muestran que las regiones genómicas que muestran divergencia evolutiva de la metilación del ADN se enriquecen para el desarrollo y especializaciones de tejidos (21). Una importante cuestión es cómo los patrones epigenéticos se transmiten entre las generaciones y la dinámica evolutiva del impacto. La fuerte correlación entre las señales evolutivas y los patrones actuales de metilación del ADN, así como la robusta conservación de la metilación génica específica del ADN en filogenias profundas, como los patrones de metilación del ADN, son evolutivamente estables para muchas regiones genómicas (21).

Liang Liu y Tollefsbol, plantean que la mayoría de los genes eucarióticos se empaquetan en las estructuras de la cromatina, lo que conduce a altas condensaciones de los genes que requieren procesos dinámicos de remodelación de la cromatina para facilitar su transcripción. La metilación del ADN y las modificaciones de las histonas representan dos de los principales procesos de remodelación de la cromatina y también sirven para integrar las señales ambientales de las células con el fin de modular la producción funcional de su genoma (16).

\section{La dieta}

Aunque es altamente especulativo, es posible que factores externos tales como la dieta puedan favorecer a la acumulación de cambios epigenéticos durante años y acelerar la manifestación de enfermedades en individuos genéticamente comprometidos (16). No solo el tipo sino la cantidad de alimento afectarían los cambios en la transcripción y traducción del ADN. Aunque, todavía se desconocen los detalles de estas alteraciones epigenéticas, se ha descubierto que los individuos de madres que vivieron periodos de hambruna tienen menor nivel de metilación en un gen implicado en el metabolismo de la insulina en comparación con sus hermanos que fueron gestados en un periodo de no hambruna (22). Además, se ha reportado que una dieta suplementada con L-metionina afecta a la metilación y la expresión de reelina. Esto es relevante porque la capacidad de reelina en los ratones reeleradores (mutante) tiene una neuropatología similar a la de la esquizofrenia (23).

El síndrome metabólico (MetS) es una combinación de trastornos médicos que aumentan el riesgo de enfermedades crónicas comunes como la obesidad, las enfermedades cardiovasculares y la diabetes. Al MetS, también se le conoce como síndrome $\mathrm{X}$, síndrome de resistencia a la insulina, síndrome de obesidad o síndrome de Reaven. Aunque el término MetS está siendo ampliamente aceptado en la literatura de investigación, todavía existe un debate en curso sobre su utilidad clínica y si califica como una condición de enfermedad para los diagnósticos clínicos $(24,25)$. La teoría de la base fetal de la enfermedad de adultos propone que los trastornos metabólicos tienen su origen en el desarrollo y están relacionados con la nutrición temprana durante la gestación y la lactancia $(16,26)$. Esta teoría ha obtenido un amplio apoyo de estudios epidemiológicos recientes que muestran que la desnutrición fetal, el bajo peso al nacer y la obesidad en humanos están asociados con un mayor riesgo de diabetes, insuficiencia cardíaca congestiva y accidente cerebrovascular (27).

Es así que, las condiciones ambientales durante el desarrollo embrionario deben ser determinadas principalmente por dos factores: 1) los fenotipos específicos de la madre y la placenta, que determinan las características, como el tamaño del útero y la disponibilidad de nutrientes; y 2) el estilo de vida de la madre, que determina los factores ambientales a los que está expuesto el embrión. Aunque poco se sabe actualmente acerca de cómo los fenotipos de la 
Epigenética: la relación del medio ambiente con el genoma y su influencia en la salud mental.

madre y la placenta pueden afectar a los epigenotipos de la descendencia (se ha sugerido que la obesidad materna puede conducir a alteraciones epigenéticas específicas de la descendencia), hay una gran cantidad de información acerca de cómo las condiciones ambientales de la madre pueden afectar a factores epigenéticos específicos de su descendencia $(25,28)$.

Oscar Aguilera y cols, discuten el posible impacto de los cambios epigenéticos en los procesos fisiológicos y fisiopatológicos, dependiendo en parte de si estos cambios ocurren durante el desarrollo embrionario o en la edad adulta. Las anormalidades del desarrollo pueden ocurrir en respuesta a la señalización epigenética inapropiada que ocurre secundariamente a causas todavía mal entendidas. Además de las influencias genéticas y estocásticas sobre los procesos epigenéticos, la variación epigenética puede surgir como consecuencia de factores ambientales (29).

\section{Las enfermedades}

Se estima que más de 8000 enfermedades humanas son causadas por defectos en genes individuales (30). Las causas de las principales enfermedades comunes, como el cáncer y la diabetes, a menudo implican genes de susceptibilidad y sus interacciones con el medio ambiente. Se cree que las interacciones genambiente mediada por modificaciones epigenéticas del genoma y los cambios epigenéticos del genoma, a menudo surgen en respuesta a los cambios en el medio ambiente (13). A diferencia de los cambios genéticos, los cambios epigenéticos son más dinámicos y a menudo son reversibles, dependiendo de la existencia o eliminación de los factores inductores. Las interacciones entre genes y el medio ambiente pueden alterar las actividades de los genes y dar lugar a cascadas de eventos celulares para facilitar la adaptación de una célula individual a su entorno. Durante mucho tiempo, se creyó que la metilación del $\mathrm{ADN}$ era el único mecanismo epigenético; sin embargo, posteriormente la remodelación de la cromatina se identificó como otro importante mecanismo epigenético a través de modificaciones postraduccionales de las proteínas histonas (16). Algunos avances recientes en el campo de la epigenética también han descubierto otros códigos epigenéticos basados en histonas tales como metilación, sumoilación, ubiquitinación, ADPribosilación y biotinilación $(13,16,31)$.

\section{Salud mental}

Bart Rutten y Jonathan Mill, resumen la evidencia actual, apoyando el papel de los procesos epigenéticos en la esquizofrenia y el trastorno bipolar y describen cómo pueden mediar la acción de los riesgos ambientales conocidos para los trastornos psicóticos principales (32). Estos aspectos son de importancia clave para la psiquiatría clínica dada la reversibilidad potencial de las marcas epigenéticas bajo la influencia de la nutrición, los factores sociales, las intervenciones conductuales y las drogas. Además de ser mitóticamente heredable, hay evidencia de que los mecanismos epigenéticos pueden ser heredables durante la meiosis en los seres humanos y, por lo tanto, potencialmente transmitidos a través de generaciones (33). Esto disuelve la demarcación entre la epigenética y secuencia de $\mathrm{ADN}$ de la herencia y desafía la suposición de que el componente "hereditario" de la psicosis y otros trastornos complejos es totalmente genético. Parece que el epigenoma es particularmente sospechoso de romperse durante una serie de períodos claves del desarrollo, especialmente durante el crecimiento prenatal cuando se está produciendo una rápida replicación celular y se están estableciendo las señales epigenéticas estándar que impulsan el desarrollo. Uno de los factores ambientales asociados con la esquizofrenia y el trastorno bipolar es la avanzada edad paterna. Se estima que menos del $1 \%$ de todos los genes están impresos por vía paterna y muchos de ellos se expresan en el cerebro, desempeñando un papel crítico en el neurodesarrollo. Curiosamente, un estudio reportó una significativa variabilidad epigenética intra e interindividual en la línea germinal masculina y encontró una serie de genes que demostraron cambios en la metilación del ADN relacionados con la edad (34). Además, reportaron en modelos celulares que las mutaciones/epimutaciones de novo pueden ser inducidas por múltiples divisiones celulares y que es una posibilidad que la exposición acumulada a diversas toxinas ambientales durante el curso de la vida pueda resultar en alteraciones de la línea germinal en hombres mayores. Dado que se ha demostrado, tales toxinas pueden inducir mutaciones en la línea germinal, daño en el ADN e hipermetilación global, es altamente plausible que tales cambios aumenten con la edad y, por tanto, que la esquizofrenia y trastorno bipolar sean más frecuente en hijos de padres mayores (35).

Además, se ha reportado evidencia de que el comportamiento y el medio ambiente influyen en la neurogénesis en el hipocampo adulto a través de mecanismos epigenéticos. De hecho, se ha demostrado que nuevas neuronas se generan en el hipocampo a lo largo de la vida, y este proceso está asociado con el aprendizaje, la memoria y el control emocional, 
cualquier efecto del medio ambiente es por lo tanto de gran importancia para nuestra comprensión de la conducta humana (36).

Es necesario destacar las maneras cómo las experiencias sociales pueden tener un efecto persistente en los procesos de diversidad fenotípica. En esta revisión, se destaca la evidencia que implica factores epigenéticos (metilación del ADN y las modificaciones de las histonas) en el vínculo entre las experiencias sociales que ocurren durante el período postnatal y en la edad adulta con los resultados neuroendocrinos y conductuales alterados (37).

La base etiológica para las formas esporádicas de las enfermedades neurodegenerativas ha sido esquiva, pero probablemente representa el producto de la predisposición genética y varios factores ambientales. El vínculo entre la lesión cerebral traumática (LCT) y la enfermedad de Parkinson (EP) es una de esas asociaciones que actualmente carece de mecanismos base. Sin embargo, Fiandaca y cols, reportan que parece haber una relación recíproca entre los niveles de ácido glutámico derivados de la sangre entre los casos y controles de LCT mínima subagudas de LCT mínima y EP. El ácido glutámico se presenta relativamente elevado en los casos de LCT en comparación con los controles. La comprensión de las relaciones entre los biomarcadores de sangre y las evaluaciones clínicas detalladas derivadas de los sujetos con LCT y EP proporcionarán un enfoque adicional para futuras investigaciones, incluidas las pistas neurobiológicas adicionales que vinculan estos distintos trastornos (38).

\section{Entorno de Crianza y Abuso Infantil}

Los estudios de adopción proporcionan evidencia de una asociación entre el ambiente de crianza, el estrés infantil y un aumento del riesgo de psicosis en el futuro. Por ejemplo, los adoptados con antecedentes familiares positivos para psicosis que habían sido educados en un entorno familiar adoptivo disfuncional tienen un mayor riesgo de desarrollar un trastorno del espectro psicótico. Aunque no se ha establecido un vínculo directo entre el trauma de la niñez, las modificaciones epigenéticas y la psicosis en los seres humanos, existe evidencia indirecta que sugiere un papel potencialmente importante para los mecanismos epigenéticos en la influencia ambiental en la psicosis. La calidad de las interacciones sociales que ocurren tempranamente en el desarrollo puede tener un profundo efecto en el desarrollo. Este efecto demuestra déficits cognitivos y sociales graves que surgen entre los niños que sufren de negligencia o abuso infantil (39-41). La interrupción de la relación padre-bebé puede conducir a deficiencias en el apego, la formación de una relación con un cuidador primario que promueve el desarrollo social y emocional, con efectos subsecuentes sobre el riesgo de la psicopatología.

Los entornos adversos prenatales, como el estrés materno, las exposiciones toxicológicas y las infecciones virales, pueden perturbar el desarrollo normal del cerebro y contribuir a trastornos del desarrollo neurológico, como la esquizofrenia, la depresión y el autismo. Los estudios en animales demuestran que la exposición prenatal al estrés, toxinas, virus y fármacos induce cambios epigenéticos duraderos en el cerebro, incluidos los genes que codifican el receptor glucocorticoide $(\mathrm{Nr} 3 \mathrm{c} 1)$ y el factor neurotrófico derivado del cerebro (Bdnf) (42). Estos cambios epigenéticos se han relacionado con cambios en la expresión de los genes cerebrales, la reactividad al estrés, el comportamiento y a menudo estos efectos se muestran dependientes de la ventana gestacional de exposición, sexo y nivel de exposición. Aunque la evidencia de los estudios en humanos es más limitada, la exposición gestacional a los riesgos ambientales en humanos está asociada con cambios epigenéticos en los tejidos periféricos y futuros estudios son necesarios para entender si podemos usar biomarcadores periféricos para predecir los resultados neurocomportamentales (42).

\section{Cannabis, abuso de otras drogas, sustancias nocivas y el genoma}

El abuso de drogas bloquea los circuitos cerebrales o afectan la funcionalidad de los circuitos cerebrales e interfieren con las funciones corporales (43). Los cannabinoides ( $\Delta 9$-tetrahidrocannabinol) forman componentes clave de la marihuana derivada de la planta de cannabis. Los mecanismos que conducen a las disparidades de salud incluyen (a) comportamientos individuales, estilo de vida, creencias y respuesta al estrés; (b) medio físico y cultural; (c) eventos clínicos y asistencia sanitaria; y (d) procesos biológicos, genéticos y epigenéticos. En general, los datos apuntan a alteraciones del epigenoma que son responsables de los efectos transcripcionales y conductuales de los efectos de larga duración de los cannabinoides, a menudo específicos del tejido (43). En los últimos años, la evidencia de estudios epidemiológicos y meta-análisis han establecido al cannabis como un claro factor de riesgo para síntomas psicóticos 
Epigenética: la relación del medio ambiente con el genoma y su influencia en la salud mental.

posteriores o trastorno psicótico. Evidencia adicional sugiere que el consumo de cannabis también se asocia con una disminución en la edad de inicio del trastorno psicótico y que las interacciones entre genes y el medio ambiente están probablemente implicadas en el consumo de cannabis (44). El componente psicoactivo primario del cannabis es el D9-tetrahidrocannabinol (THC), que se cree que ejerce sus efectos psicológicos a través de la interrupción de la señalización mediada por el receptor de cannabis-1 normal en el cerebro. La administración de THC o cannabis provoca cambios moleculares y celulares a largo plazo en los cerebros de ratones y seres humanos. El abuso de otras drogas psicoestimulantes, como la cocaína, la anfetamina y la fenciclidina, también se ha asociado sistemáticamente con trastornos psicóticos importantes; los pacientes con trastornos psicóticos mayores frecuentemente usan, abusan y se vuelven dependientes de estas drogas (45). Además, los fármacos psicoestimulantes son bien conocidos por provocar síntomas psicóticos en individuos sin esquizofrenia o trastorno bipolar. La creciente evidencia de los análisis de casos y controles de alteraciones epigenéticas y los hallazgos de estudios epidemiológicos complementados con trabajos experimentales en animales sugieren un papel de la maquinaria epigenética de las células en la mediación de los efectos de las exposiciones ambientales en la etiología de los trastornos psicóticos principales (32). Las alteraciones epigenéticas más estudiadas por el abuso de drogas son las causadas por la cocaína. Se sabe que la administración materna de cocaína en ratones altera la metilación del ADN y la expresión génica en neuronas del hipocampo de descendientes neonatales y pre-púberes (46). Además, la regulación de genes por el HMT G9a tiene un papel esencial en la plasticidad inducida por la cocaína y los inhibidores de HDAC disminuyen la auto-administración de cocaína en ratas (47). Estas observaciones sugieren que una serie de efectos fisiológicos de la cocaína in vivo están mediados por mecanismos epigenéticos. Se necesitan más investigaciones para determinar el impacto real del abuso de otros fármacos en el epigenoma del organismo. Aunque es evidente que la mayoría de las sustancias descritas anteriormente pueden alterar factores epigenéticos, debe destacarse que no se sabe si todos ellos pueden ser considerados auténticos modificadores epigenéticos, porque aún no se ha demostrado si las modificaciones epigenéticas que ellos inducen son estables con el tiempo.

Por otro lado, se ha propuesto que el dietilestilbestrol, un fármaco utilizado por millones de mujeres embarazadas para prevenir los abortos espontáneos y muchos otros trastornos en el embarazo, que actualmente se sabe que está asociado con un mayor riesgo de cáncer de mama, adenocarcinoma de células claras de la vagina y el cuello uterino, puede estar mediado por mecanismos epigenéticos, ya que se ha demostrado que este fármaco altera la expresión de las metiltransferasas del ADN y la metilación del ADN genómico $(43,48)$.

\section{CONCLUSIONES}

La epigenética abarca todos los mecanismos implicados en el despliegue del programa genético para los muchos procesos que operan durante la vida útil de una célula. Aunque las modificaciones epigenéticas parecen ser estables, pueden ser moduladas por muchos factores, incluyendo las condiciones fisiológicas, patológicas y/o por el medio ambiente. Para entender el impacto del ambiente en el epigenotipo, es necesario considerar dos escenarios: el desarrollo embrionario y la vida adulta. En principio, los cambios epigenéticos que ocurren durante el desarrollo embrionario tendrán un impacto mucho mayor en el estado epigenético global del organismo en la vida adulta ya que pueden ser transmitidos a través de divisiones mitóticas consecutivas. Las alteraciones ocurridas en células madre embrionarias individuales afectarán a muchas más células que en las células adultas del tallo y/o somáticas durante el desarrollo postnatal. En el ser humano, los factores ambientales que pueden afectar el estado epigenético durante la vida adulta pueden dividirse en cuatro grupos: dieta, lugar de residencia y/o lugar de trabajo, tratamientos farmacológicos y hábitos no saludables. Aunque parece obvio que, si los factores epigenéticos pueden ser alterados por los compuestos de la dieta, entonces también deberían ser susceptibles de alteración por sustancias farmacológicas; la evidencia para esto último aún es limitada.

\section{Correspondencia:}

Sandro Casavilca-Zambrano

Correo electrónico: scasavilca@inen.sld.pe

\section{REFERENCIAS BIBLIOGRÁFICAS}

1. Hood L, Rowen L. The human genome project: big science transforms biology and medicine. Genome Med. 2013;5(9):79.

2. Wong AHC, Gottesman II, Petronis A. Phenotypic differences in genetically identical organisms: the epigenetic perspective. Hum Mol Genet. 2005;14 
(suppl_1):R11-8. DOI: $10.1093 / \mathrm{hmg} / \mathrm{ddi} 116$

3. Almouzni G, Altucci L, Amati B, et al. Relationship between genome and epigenome - challenges and requirements for future research. BMC Genomics. 2014;15(1):487. doi: 10.1186/1471-2164-15-487

4. Purrucker JC, Mahlknecht U. Targeting the epigenome: effects of epigenetic treatment strategies on genomic stability in healthy human cells. Clin Epigenetics. 2010; 1(1-2):45-54.

5. Jones PA, Baylin SB. The epigenomics of cancer. Cell. 2007;128(4):683-92.

6. Yoo CB, Jones PA. Epigenetic therapy of cancer: past, present and future. Nat Rev Drug Discov. 2006;5(1):37-50.

7. Biémont C. From genotype to phenotype. What do epigenetics and epigenomics tell us? Heredity (Edinb). 2010;105(1):1-3.

8. Johannes F, Porcher E, Teixeira FK, et al. Assessing the Impact of Transgenerational Epigenetic Variation on Complex Traits. PLoS Genet. 2009;5(6):e1000530.

9. Johannes F, Colot V, Jansen RC. Epigenome dynamics: a quantitative genetics perspective. Nat Rev Genet. 2008;9(11):883-90.

10. Riggs AD. $X$ inactivation, differentiation, and DNA methylation. Cytogenet Genome Res. 1975;14(1):925.

11. Holliday R, Pugh JE. DNA modification mechanisms and gene activity during development. Science. 1975;187(4173):226-32.

12. Meyers R. Synthetic biology. Weinheim: Wiley VCH; 2006.

13. Jaenisch R, Bird A. Epigenetic regulation of gene expression: how the genome integrates intrinsic and environmental signals. Nat Genet. 2003;33(S3):24554.

14. Bianchi M, Renzini A, Adamo S, Moresi V. Coordinated Actions of MicroRNAs with other epigenetic factors regulate skeletal muscle development and adaptation. Int $\mathrm{J}$ Mol Sci. 2017;18(4):0-0. doi: 10.3390/ijms18040840

15. Ordog T, Syed SA, Hayashi Y, Asuzu DT. Epigenetics and chromatin dynamics: a review and a paradigm for functional disorders. Neurogastroenterol Motil. 2012;24(12):1054-68.

16. Liu L, Li Y, Tollefsbol TO. Gene-environment interactions and epigenetic basis of human diseases. Curr Issues Mol Biol. 2008; 10(1-2):25-36.

17. Goldberg AD, Allis CD, Bernstein E. Epigenetics: A Landscape Takes Shape. Cell. 2007;128(4):635-8.

18. Cheung P, Lau P. Epigenetic Regulation by Histone Methylation and Histone Variants. Mol Endocrinol. 2005;19(3):563-73.

19. Hatada I. The Epigenomics of Cancer. In: Omenn G. An Omics Perspective on Cancer Research. Dordrecht: Springer Netherlands; 2010. p. 51-67.

20. Nan X, Ng H-H, Johnson CA, et al. Transcriptional repression by the methyl-CpG-binding protein $\mathrm{MeCP} 2$ involves a histone deacetylase complex. Nature. 1998;393(6683):386-9.

21. Mendizabal I, Keller TE, Zeng J, Yi S V. Epigenetics and Evolution. Integr Comp Biol. 2014;54(1):31-42.

22. Heijmans BT, Tobi EW, Stein AD, et al. Persistent epigenetic differences associated with prenatal exposure to famine in humans. Proc Natl Acad Sci. 2008;105(44):17046-9.

23. Dong E, Agis-Balboa RC, Simonini M V., Grayson DR, Costa E, Guidotti A. Reelin and glutamic acid decarboxylase 67 promoter remodeling in an epigenetic methionine-induced mouse model of schizophrenia. Proc Natl Acad Sci. 2005;102(35):12578-83.

24. Sales VM, Ferguson-Smith AC, Patti M-E. Epigenetic Mechanisms of Transmission of Metabolic Disease across Generations. Cell Metab. 2017;25(3):559-71.

25. Gallou-Kabani C, Junien C. Nutritional Epigenomics of Metabolic Syndrome: New Perspective Against the Epidemic. Diabetes. 2005;54(7):1899-906.

26. Grundy SM. Obesity, metabolic syndrome, and cardiovascular disease. J Clin Endocrinol Metab. 2004;89(6):2595-600.

27. Matfin G. The metabolic syndrome: what's in a name? Ther Adv Endocrinol Metab. 2010;1(2):3945.

28. Bihaqi SW. Early life exposure to lead $(\mathrm{Pb})$ and changes in DNAmethylation: relevance to Alzheimer's disease. Rev Environ Health. 2019;34(2):187-95.

29. Aguilera O, Fernández AF, Muñoz A, Fraga MF. Epigenetics and environment: a complex relationship. J Appl Physiol. 2010;109(1):243-51.

30. Kaplan J. Genomics and medicine: hopes and challenges. Gene Ther. 2002;9:658-61.

31. Hassan YI, Zempleni J. Epigenetic regulation of chromatin structure and gene function by biotin. J Nutr. 2006; 136(7):1763-5.

32. Rutten BPF, Mill J. Epigenetic mediation of environmental influences in major psychotic disorders. Schizophr Bull. 2009;35(6):1045-56.

33. Grossniklaus U, Kelly WG, Kelly B, FergusonSmith AC, Pembrey M, Lindquist S. Transgenerational epigenetic inheritance: how important is it? Nat Rev Genet. 2013; 14(3):228-35.

34. Flanagan JM, Popendikyte V, Pozdniakovaite N, et al. Intra- and Interindividual Epigenetic Variation in Human Germ Cells. Am J Hum Genet. 2006;79(1):6784.

35. Milekic MH, Xin Y, O'Donnell A, et al. Age-related sperm DNA methylation changes are transmitted to offspring and associated with abnormal behavior and dysregulated gene expression. Mol Psychiatry. 2015;20(8):995-1001.

36. Ihunwo A, Tembo L, Dzamalala C. The dynamics of adult neurogenesis in human hippocampus. Neural Regen Res. 2016;11(12):1869. 
Epigenética: la relación del medio ambiente con el genoma y su influencia en la salud mental.

37. Champagne FA. Interplay Between Social Experiences and the Genome: Epigenetic Consequences for Behavior. In: Kumar D. Advances in genetics. 2012; Cardiff: Cardiff University. p. 3357.

38. Fiandaca MS, Gross TJ, Johnson TM, Hu MT, Evetts S, Wade-Martins R, et al. Potential Metabolomic Linkage in Blood between Parkinson's Disease and Traumatic Brain Injury. Metabolites. 2018;8:50.

39. Eluvathingal TJ, Chugani HT, Behen ME, Juhász C, Muzik O, Maqbool M, et al. Abnormal Brain Connectivity in Children After Early Severe Socioemotional Deprivation: A Diffusion Tensor Imaging Study. Pediatrics. 2006;117(6):2093-100.

40. MacLean CD, Susi B, Phifer N, Schultz L, Bynum D, Franco M, et al. Patient preference for physician discussion and practice of spirituality. J Gen Intern Med. 2003;18(1):38-43.

41. Trickett PK, McBride-Chang C. The Developmental Impact of Different Forms of Child Abuse and Neglect. Dev Rev. 1995;15(3):311-37.

42. Kundakovic M, Jaric I. The epigenetic link between prenatal adverse environments and neurodevelopmental disorders. Genes (Basel). 2017;8(3):104.
43. Heinbockel T, Csoka AB. Epigenetic Effects of Drugs of Abuse. Int J Environ Res Public Health. 2018;15(10):2098.

44. Wilkinson ST, Radhakrishnan R, D'Souza DC. Impact of Cannabis Use on the Development of Psychotic Disorders. Curr Addict reports. 2014;1(2):115-28.

45. Bowers M, Jr., Boutros N, D’Souza DC, Madonick S. Substance Abuse as a Risk Factor for Schizophrenia and Related Disorders. International Journal of Mental Health. 2001; 30: 33-57.

46. Novikova SI, He F, Bai J, Cutrufello NJ, Lidow MS, Undieh AS. Maternal Cocaine Administration in Mice Alters DNA Methylation and Gene Expression in Hippocampal Neurons of Neonatal and Prepubertal Offspring. PLoS One. 2008;3(4):e1919.

47. Berkel TDM, Zhang H, Teppen T, Sakharkar AJ, PandeySC.EssentialRoleofHistoneMethyltransferase G9a in Rapid Tolerance to the Anxiolytic Effects of Ethanol. Int J Neuropsychopharmacol. 2019;22(4):292-302.

48. Mews P, Walker DM, Nestler EJ. Epigenetic Priming in Drug Addiction. Cold Spring Harb Symp Quant Biol. 2019;037663.

Recibido: 05/11/2019

Aceptado: 07/12/2019 